Supplement of Biogeosciences, 15, 3857-3871, 2018

https://doi.org/10.5194/bg-15-3857-2018-supplement

(C) Author(s) 2018. This work is distributed under

the Creative Commons Attribution 4.0 License.

(c) (1)

Supplement of

\title{
Riverine carbon export in the arid to semiarid Wuding River catchment on the Chinese Loess Plateau
}

\section{Lishan Ran et al.}

Correspondence to: Lishan Ran (ranlishan@gmail.com)

The copyright of individual parts of the supplement might differ from the CC BY 4.0 License. 


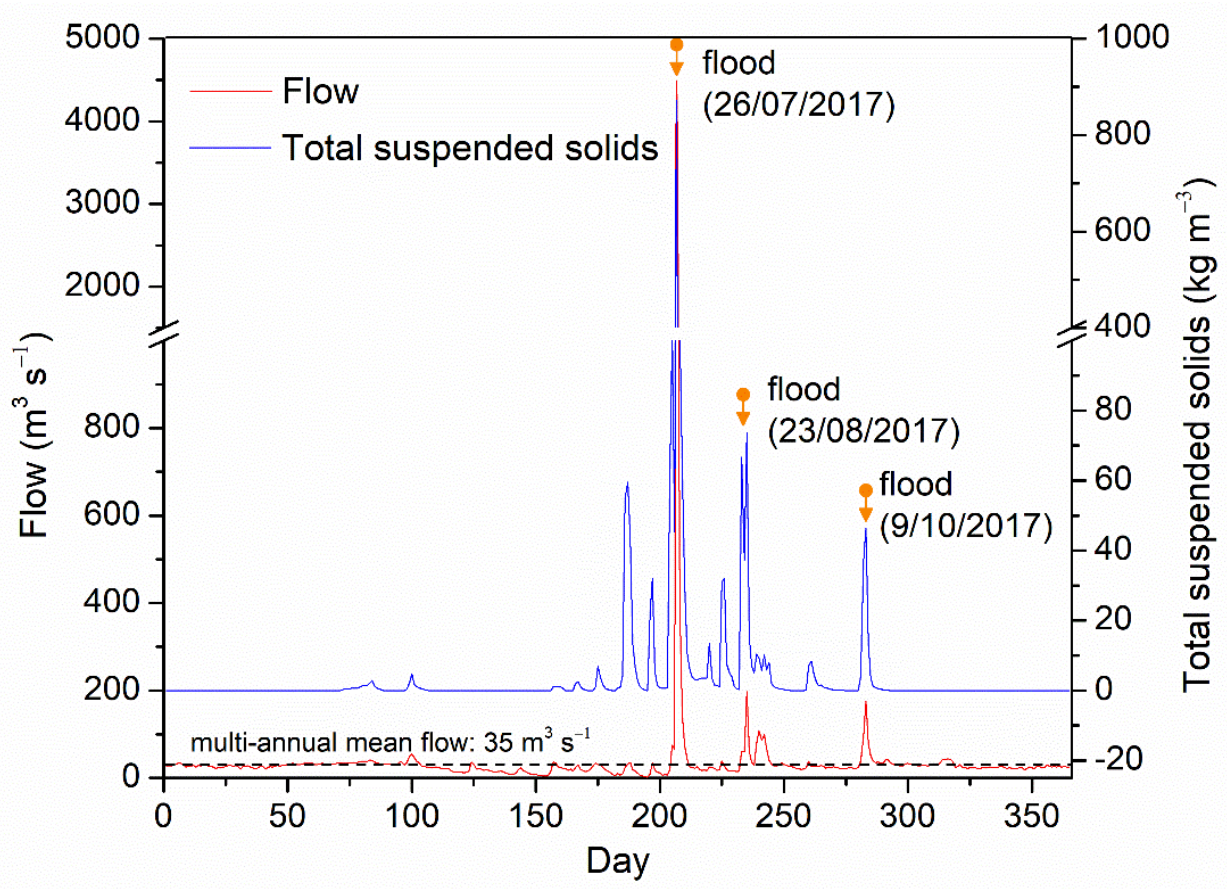

Figure S1. Spontaneous flow discharge and total suspended solids in the Wuding River at Baijiachuan gauge in 2017. The duration started from 1 January until 31 December 2017. The extreme flood on 25-26 July with the maximum daily rainfall of $203 \mathrm{~mm}$ and a spontaneous discharge of $4490 \mathrm{~m}^{3} \mathrm{~s}^{-1}$ is a 200 -year event (source: http://www.sxmb.gov.cn/s.php/yulin). Our conservative estimate suggests that the three floods (shown in the figure) in the wet season transported approximately $46 \%$ of the annual carbon flux.

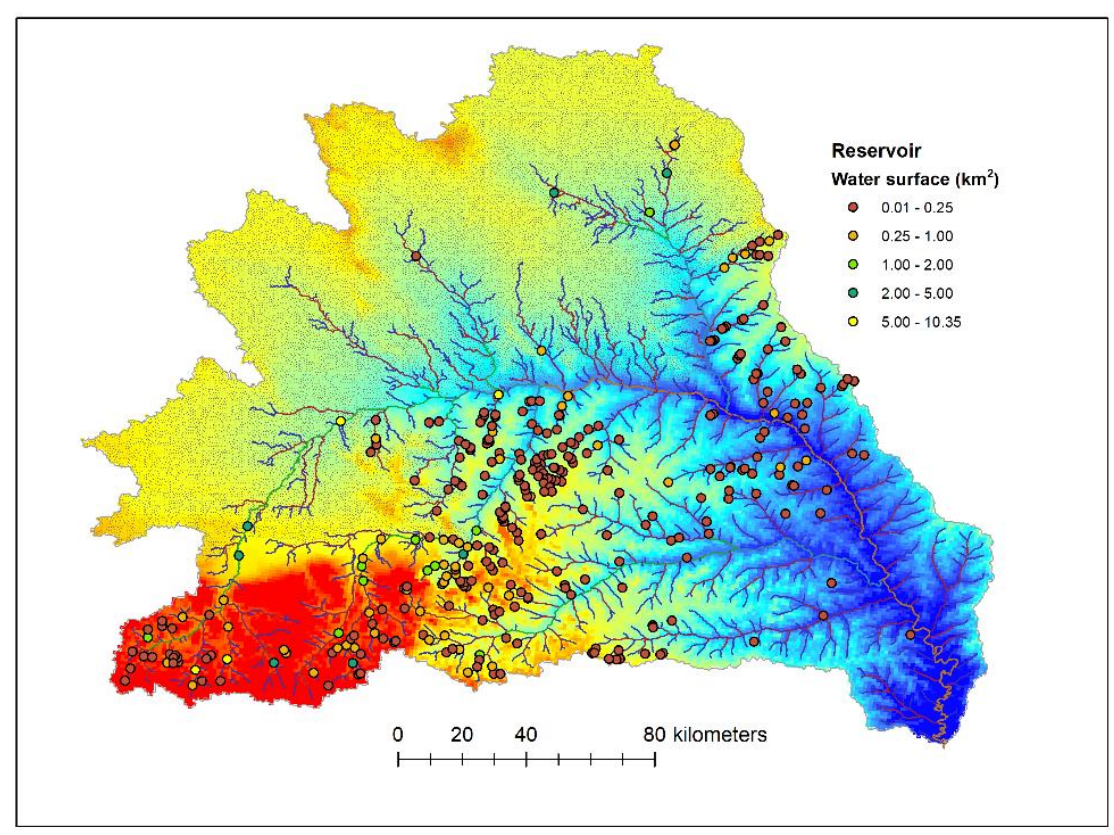

Figure S2. Spatial location of the 337 check dam-formed reservoirs within the Wuding River catchment. 
Table S1. POC\% in suspended sediments (dry weight) in spring, summer, and autumn within the Wuding River catchment in 2015 (n.d. denotes no data).

\begin{tabular}{|c|c|c|c|c|c|c|c|}
\hline Site & $\mathbf{E}\left({ }^{\circ}\right)$ & $\mathbf{N}\left({ }^{\circ}\right)$ & $\begin{array}{l}\text { POC\% } \\
\text { (spring/summer/autumn) }\end{array}$ & Site & $\mathbf{E}\left({ }^{\circ}\right)$ & $\mathbf{N}\left({ }^{\circ}\right)$ & $\begin{array}{l}\text { POC\% } \\
\text { (spring/summer/autumn) }\end{array}$ \\
\hline Site 1 & 109.6704 & 38.7150 & $0.52 / 0.44 / 0.57$ & Site 31 & 109.202911 & 37.731611 & $1.72 / 0.38 / 0.45$ \\
\hline Site 2 & 109.6519333 & 38.5394833 & $0.59 /$ n.d. $/ 0.51$ & Site 32 & 109.913062 & 37.723534 & $1.14 / 0.51 / 0.65$ \\
\hline Site 3 & 109.572779 & 38.59416 & $0.37 / 0.29 / 0.48$ & Site 33 & 109.642591 & 38.531045 & n.d. / 0.44 / 0.88 \\
\hline Site 4 & 109.586677 & 38.616434 & $0.78 / 0.42 / 0.65$ & Site 34 & 109.761716 & 37.651897 & n.d. / $0.41 / 0.44$ \\
\hline Site 5 & 109.280033 & 37.91626 & $0.84 / 0.57 / 0.82$ & Site 35 & 109.574101 & 38.50211 & $0.71 / 0.39 / 0.84$ \\
\hline Site 6 & 109.204611 & 37.756443 & $1.05 / 0.75 / 0.58$ & Site 36 & 109.620762 & 38.488028 & $0.31 / 0.37 / 0.45$ \\
\hline Site 7 & 109.204482 & 37.755505 & $0.88 / 0.38 / 0.55$ & Site 37 & 109.015674 & 38.036713 & $0.49 / 0.42 / 0.28$ \\
\hline Site 8 & 109.12282 & 37.691243 & $0.74 / 0.53 / 0.42$ & Site 38 & 109.019899 & 38.023019 & $0.81 / 0.36 / 0.87$ \\
\hline Site 9 & 109.963279 & 37.657502 & $1.35 / 0.72 / 0.64$ & Site 39 & 109.044785 & 37.963968 & $0.95 / 0.43 / 0.74$ \\
\hline Site 10 & 109.934874 & 37.688188 & $0.89 / 0.47 / 0.65$ & Site 40 & 109.170892 & 38.096652 & $0.64 / 0.31 / 0.53$ \\
\hline Site 11 & 109.929881 & 37.707845 & $1.14 / 0.49 / 0.78$ & Site 41 & 109.848753 & 37.604823 & $0.96 / 0.38 / 0.81$ \\
\hline Site 12 & 109.8936667 & 37.762965 & $1.37 / 0.51 / 0.77$ & Site 42 & 109.535868 & 37.555864 & $0.79 / 0.37 / 0.92$ \\
\hline Site 13 & 109.843565 & 38.105545 & n.d. / $0.58 / 0.41$ & Site 43 & 109.788402 & 37.627569 & $0.87 / 0.46 / 0.98$ \\
\hline Site 14 & 109.6684 & 38.7134 & $0.88 / 0.49 / 0.46$ & Site 44 & 109.677892 & 38.4444 & $0.65 / 0.39 / 1.02$ \\
\hline Site 15 & 109.586627 & 38.585814 & $0.93 / 0.50 / 0.70$ & Site 45 & 109.770647 & 38.128133 & $0.92 / 0.36 / 0.78$ \\
\hline Site 16 & 109.033164 & 37.962789 & $0.56 / 0.46 / 0.79$ & Site 46 & 109.82468 & 38.000788 & $1.25 / 0.39 / 0.58$ \\
\hline Site 17 & 109.033164 & 37.962789 & $1.28 / 0.59 / 0.76$ & Site 47 & 109.068546 & 38.020439 & n.d. / $0.41 / 0.64$ \\
\hline Site 18 & 109.208739 & 37.719403 & $0.41 /$ n.d. / 0.68 & Site 48 & 109.201555 & 37.803375 & $0.63 / 0.29 / 0.68$ \\
\hline Site 19 & 109.212468 & 37.714357 & $0.49 / 0.65 / 0.85$ & Site 49 & 109.152867 & 37.739736 & $1.35 / 0.38 / 0.63$ \\
\hline Site 20 & 109.872779 & 37.745172 & $0.84 / 0.55 / 0.91$ & Site 50 & 109.12282 & 37.691243 & $0.98 / 0.49 / 0.59$ \\
\hline Site 21 & 109.902658 & 37.737476 & $0.93 / 0.52 / 0.74$ & Site 51 & 110.001214 & 37.625839 & 1.17 / n.d. / 0.67 \\
\hline Site 22 & 109.896515 & 37.747859 & $0.76 / 0.31 / 0.56$ & Site 52 & 109.963279 & 37.657502 & $0.89 / 0.36 / 1.42$ \\
\hline Site 23 & 109.811722 & 38.083644 & n.d. / $0.33 / 0.54$ & Site 53 & 109.758937 & 38.02164 & $0.62 / 0.36 / 0.55$ \\
\hline Site 24 & 109.843565 & 38.105545 & n.d. / $0.37 / 0.48$ & Site 54 & 109.617308 & 38.034026 & $0.88 / 0.44 / 0.82$ \\
\hline Site 25 & 109.6423 & 38.5111 & $0.56 / 0.43 / 0.42$ & Site 55 & 109.991145 & 37.951565 & $1.36 / 0.55 / 0.98$ \\
\hline Site 26 & 109.657208 & 38.493477 & $0.94 / 0.44 / 0.50$ & Site 56 & 110.136182 & 37.80883 & $1.34 / 0.46 / 0.84$ \\
\hline Site 27 & 109.633408 & 38.507959 & $0.78 / 0.37 / 1.14$ & Site 57 & 110.307521 & 37.446976 & $1.60 / 0.41 / 1.18$ \\
\hline Site 28 & 109.50634 & 38.513226 & $0.88 / 0.39 / 0.64$ & Site 58 & 110.364369 & 37.374946 & $1.66 / 0.48 / 0.58$ \\
\hline Site 29 & 109.617308 & 38.034026 & $0.79 / 0.58 / 0.61$ & Site 59 & 110.405976 & 37.294277 & $1.08 / 0.45 / 0.51$ \\
\hline Site 30 & 109.186599 & 37.769123 & $0.70 / 0.47 / 1.04$ & Site 60 & 110.46104 & 37.27201 & n.d. / 0.35 / n.d. \\
\hline
\end{tabular}


Table S2. Monthly carbon sampling results at the Wuding River catchment outlet Baijiachuan gauge in 2017. Note: the daily average discharge in 2015 on the same days as in 2017 is also presented here (n.d. denotes no data).

\begin{tabular}{|l|l|l|l|l|l|}
\hline \multirow{2}{*}{$\begin{array}{l}\text { Date } \\
(\mathrm{mm} / \mathrm{dd})\end{array}$} & \multicolumn{2}{|l|}{ Flow discharge $\left.\mathbf{~ m}^{\mathbf{3}} / \mathbf{s}\right)$} & DOC & DIC & POC \\
\cline { 2 - 6 } & Year 2015 & Year 2017 & $\mathrm{mg} / \mathrm{L}$ & $\mathrm{mg} / \mathrm{L}$ & $\%$ \\
\hline $01 / 20$ & 25 & 24.5 & 2.66 & 36 & 0.47 \\
\hline $02 / 20$ & 34.3 & 32.5 & 2.9 & 31.2 & 0.62 \\
\hline $03 / 20$ & 40.1 & 36.6 & 3.55 & 41.4 & 0.69 \\
\hline $04 / 20$ & 26.4 & 24.6 & 3.54 & 42 & 0.64 \\
\hline $05 / 20$ & 11.5 & 8.23 & 3.68 & 42.6 & 0.68 \\
\hline $06 / 20$ & 5.25 & 16.0 & 3.66 & 37.8 & 1.16 \\
\hline $07 / 22$ & 50.2 & 6.85 & 3.51 & 46.2 & 0.64 \\
\hline $07 / 26$ & 11.6 & 1760.0 & 5.22 & n.d. & 0.15 \\
\hline $08 / 23$ & 17.6 & 198.0 & 5.07 & 46.8 & 0.46 \\
\hline $09 / 20$ & 25.8 & 26.6 & 3.47 & 33.6 & 0.72 \\
\hline $10 / 20$ & 23.9 & 34.5 & 2.9 & 39 & 0.49 \\
\hline $11 / 20$ & 49.4 & 22.2 & 2.79 & 34.2 & 0.53 \\
\hline $12 / 20$ & 19.6 & 23.2 & 2.73 & 36.6 & 0.69 \\
\hline
\end{tabular}

Table S3. Sediment POC\% (dry weight) behind the four investigated check dams within the Wuding River catchment.

\begin{tabular}{|l|l|l|l|l|l|l|l|}
\hline \multicolumn{2}{|c|}{ Site S1 } & \multicolumn{2}{c|}{ Site S2 } & \multicolumn{2}{c|}{ Site S3 } & \multicolumn{2}{c|}{ Site S4 } \\
\hline E: $109.5921^{\circ}$ & \multicolumn{1}{c|}{ E: $109.0996^{\circ}$} & \multicolumn{2}{c|}{ E: $109.8397^{\circ}$} & \multicolumn{1}{l|}{ E: $109.1789^{\circ}$} \\
N: $38.56712^{\circ}$ & N: $37.61303^{\circ}$ & N: $37.58147^{\circ}$ & N: $37.87671^{\circ}$ \\
\hline $0 \mathrm{~cm}$ & 0.255 & $12.5 \mathrm{~cm}$ & 0.306 & $0 \mathrm{~cm}$ & 0.443 & $12.5 \mathrm{~cm}$ & 0.438 \\
\hline $20 \mathrm{~cm}$ & 0.505 & $37.5 \mathrm{~cm}$ & 0.139 & $20 \mathrm{~cm}$ & 0.503 & $37.5 \mathrm{~cm}$ & 0.366 \\
\hline $40 \mathrm{~cm}$ & 0.225 & $62.5 \mathrm{~cm}$ & 0.187 & $40 \mathrm{~cm}$ & 0.495 & $62.5 \mathrm{~cm}$ & 0.483 \\
\hline $60 \mathrm{~cm}$ & 0.155 & $87.5 \mathrm{~cm}$ & 0.151 & $60 \mathrm{~cm}$ & 0.113 & $87.5 \mathrm{~cm}$ & 0.506 \\
\hline $80 \mathrm{~cm}$ & 0.385 & $112.5 \mathrm{~cm}$ & 0.13 & $80 \mathrm{~cm}$ & 0.112 & $112.5 \mathrm{~cm}$ & 0.277 \\
\hline $100 \mathrm{~cm}$ & 0.12 & $137.5 \mathrm{~cm}$ & 0.226 & $100 \mathrm{~cm}$ & 0.109 & $137.5 \mathrm{~cm}$ & 0.367 \\
\hline $120 \mathrm{~cm}$ & 0.175 & $162.5 \mathrm{~cm}$ & 0.117 & $120 \mathrm{~cm}$ & 0.12 & $162.5 \mathrm{~cm}$ & 0.307 \\
\hline $140 \mathrm{~cm}$ & 0.175 & $187.5 \mathrm{~cm}$ & 0.152 & $140 \mathrm{~cm}$ & 0.08 & $187.5 \mathrm{~cm}$ & 0.339 \\
\hline $160 \mathrm{~cm}$ & 0.155 & $212.5 \mathrm{~cm}$ & 0.151 & $160 \mathrm{~cm}$ & 0.105 & $212.5 \mathrm{~cm}$ & 0.231 \\
\hline $180 \mathrm{~cm}$ & 0.13 & $237.5 \mathrm{~cm}$ & 0.15 & $180 \mathrm{~cm}$ & 0.2 & $237.5 \mathrm{~cm}$ & 0.178 \\
\hline $200 \mathrm{~cm}$ & 0.163 & $262.5 \mathrm{~cm}$ & 0.134 & $200 \mathrm{~cm}$ & 0.11 & $262.5 \mathrm{~cm}$ & 0.142 \\
\hline $220 \mathrm{~cm}$ & 0.197 & $287.5 \mathrm{~cm}$ & 0.128 & $220 \mathrm{~cm}$ & 0.1 & $287.5 \mathrm{~cm}$ & 0.177 \\
\hline $240 \mathrm{~cm}$ & 0.183 & $312.5 \mathrm{~cm}$ & 0.119 & $240 \mathrm{~cm}$ & 0.14 & $312.5 \mathrm{~cm}$ & 0.266 \\
\hline $260 \mathrm{~cm}$ & 0.21 & $337.5 \mathrm{~cm}$ & 0.159 & $260 \mathrm{~cm}$ & 0.125 & $337.5 \mathrm{~cm}$ & 0.208 \\
\hline $280 \mathrm{~cm}$ & 0.164 & $362.5 \mathrm{~cm}$ & 0.181 & $280 \mathrm{~cm}$ & 0.13 & $362.5 \mathrm{~cm}$ & 0.26 \\
\hline $300 \mathrm{~cm}$ & 0.156 & $387.5 \mathrm{~cm}$ & 0.121 & $300 \mathrm{~cm}$ & 0.14 & $387.5 \mathrm{~cm}$ & 0.225 \\
\hline $320 \mathrm{~cm}$ & 0.18 & $412.5 \mathrm{~cm}$ & 0.087 & $320 \mathrm{~cm}$ & 0.14 & $412.5 \mathrm{~cm}$ & 0.234 \\
\hline $340 \mathrm{~cm}$ & 0.188 & $437.5 \mathrm{~cm}$ & 0.115 & $340 \mathrm{~cm}$ & 0.1 & $437.5 \mathrm{~cm}$ & 0.255 \\
\hline $360 \mathrm{~cm}$ & 0.165 & $462.5 \mathrm{~cm}$ & 0.113 & $360 \mathrm{~cm}$ & 0.125 & $462.5 \mathrm{~cm}$ & 0.209 \\
\hline $380 \mathrm{~cm}$ & 0.213 & $487.5 \mathrm{~cm}$ & 0.221 & $380 \mathrm{~cm}$ & 0.11 & $487.5 \mathrm{~cm}$ & 0.213 \\
\hline $400 \mathrm{~cm}$ & 0.167 & $512.5 \mathrm{~cm}$ & 0.103 & $400 \mathrm{~cm}$ & 0.115 & $512.5 \mathrm{~cm}$ & 0.281 \\
\hline & & $537.5 \mathrm{~cm}$ & 0.139 & & & $537.5 \mathrm{~cm}$ & 0.245 \\
\hline & & $562.5 \mathrm{~cm}$ & 0.195 & & & $562.5 \mathrm{~cm}$ & 0.259 \\
\hline & & $587.5 \mathrm{~cm}$ & 0.124 & & & $587.5 \mathrm{~cm}$ & 0.122 \\
\hline
\end{tabular}


Table S4. Water surface area of the Wuding River drainage network (expressed as mean \pm standard deviation; SD).

\begin{tabular}{|c|c|c|c|c|c|c|c|c|c|c|}
\hline \multirow{2}{*}{$\begin{array}{c}\text { Stream } \\
\text { order } \\
\text { (SO) }\end{array}$} & \multicolumn{3}{|c|}{ Sandy subcatchment } & \multicolumn{3}{|c|}{ Loess subcatchment } & \multicolumn{4}{|c|}{ Wuding catchment } \\
\hline & $\begin{array}{c}\text { Spring } \\
\left(\mathrm{km}^{2}\right)\end{array}$ & $\begin{array}{c}\text { Summer } \\
\left(\mathrm{km}^{2}\right)\end{array}$ & $\begin{array}{c}\text { Autumn } \\
\left(\mathrm{km}^{2}\right)\end{array}$ & $\begin{array}{l}\text { Spring } \\
\left(\mathrm{km}^{2}\right)\end{array}$ & $\begin{array}{c}\text { Summer } \\
\left(\mathrm{km}^{2}\right)\end{array}$ & $\begin{array}{c}\text { Autumn } \\
\left(\mathrm{km}^{2}\right)\end{array}$ & $\begin{array}{c}\text { Spring } \\
\left(\mathrm{km}^{2}\right)\end{array}$ & $\begin{array}{c}\text { Summer } \\
\left(\mathrm{km}^{2}\right)\end{array}$ & $\begin{array}{c}\text { Autumn } \\
\left(\mathrm{km}^{2}\right)\end{array}$ & \%total \\
\hline 1 & $2.33 \pm 1.38$ & $2.33 \pm 1.36$ & $2.5 \pm 0.96$ & $6.63 \pm 4.33$ & $5.05 \pm 4.61$ & $5.63 \pm 4.41$ & $8.93 \pm 5.67$ & $7.38 \pm 5.83$ & $8.12 \pm 5.26$ & $20 \%$ \\
\hline 2 & $1.7 \pm 0.77$ & $1.33 \pm 0.98$ & $2.48 \pm 2.05$ & $2.65 \pm 0.87$ & $1.73 \pm 0.82$ & $3.04 \pm 1.52$ & $4.2 \pm 1.53$ & $2.83 \pm 1.59$ & $5.06 \pm 3.14$ & $10 \%$ \\
\hline 3 & $1.45 \pm 1.1$ & $1.37 \pm 0.96$ & $2.15 \pm 1.98$ & $1.14 \pm 0.14$ & $1.84 \pm 1.56$ & $1.28 \pm 0.5$ & $2.8 \pm 2.11$ & $3.26 \pm 2.38$ & $3.81 \pm 3.88$ & $8 \%$ \\
\hline 4 & $1.45 \pm 0.82$ & $1.39 \pm 0.95$ & $1.38 \pm 0.29$ & $2.51 \pm 0.07$ & $2.19 \pm 1.32$ & $1.6 \pm 0.92$ & $3.7 \pm 1.65$ & $3.5 \pm 2.13$ & $3.04 \pm 1.12$ & $9 \%$ \\
\hline 5 & $0.55 \pm 0.09$ & $0.78 \pm 0.03$ & $0.79 \pm 0.07$ & $3.11 \pm 3.36$ & $3.2 \pm 4.42$ & $3.13 \pm 4.06$ & $3.77 \pm 4.12$ & $4.01 \pm 5.18$ & $4 \pm 4.88$ & $10 \%$ \\
\hline 6 & 5.03 & 5.01 & 4.79 & $13.9 \pm 4.94$ & $10.69 \pm 7.84$ & $12.41 \pm 4.99$ & $18.88 \pm 8.66$ & $15.24 \pm 11.47$ & $16.82 \pm 8.42$ & $43 \%$ \\
\hline Total & \multicolumn{3}{|c|}{12.9} & \multicolumn{3}{|c|}{27.2} & \multicolumn{3}{|c|}{40.1} & \\
\hline \%total & \multicolumn{3}{|c|}{$32 \%$} & \multicolumn{3}{|c|}{$68 \%$} & \multicolumn{3}{|c|}{$100 \%$} & \\
\hline
\end{tabular}

